\title{
COVID-19 in a child with down syndrome and extensive pulmonary tuberculosis: a case report
}

Pedro Pollini Gonçalves Stefanuto 1

iD https://orcid.org/0000-0001-7258-2677

Carla Jéssica da Silva Fernandes 2

iD https://orcid.org/0000-0003-4240-1921

Camilla Gomes da Cruz 3

iD https://orcid.org/0000-0003-2649-4224

Robério Dias Leite 4

iD https://orcid.org/0000-0003-4416-0543

Lohanna Valeska de Sousa Tavares 5

(iD) https://orcid.org/0000-0002-6426-0755

1,2 Enfermaria de Pediatria Geral. Hospital Geral de Fortaleza. Rua Ávila Goulart, 900. Bairro Papicu. Fortaleza, CE, Brasil. CEP: 60.150-160. E-mail: pedropollini17@gmail.com

3 Enfermaria de Pediatria Geral. Hospital Infantil Albert Sabin. Fortaleza, CE, Brasil.

4,5 Hospital São José de Doenças Infecciosas. Fortaleza, CE, Brasil.

\begin{abstract}
Introduction: although pediatric patients have comparatively fewer cases of COVID-19, children with Down Syndrome exhibit comorbidities such as immunodeficiency, diabetes and, in this perspective, are considered a population at risk for severe COVID-19. In addition, the literature also points to an unfavorable perspective on co-infection with Mycobacterium tuberculosis, considered an important comorbidity and a predictor of a worse clinical outcome.

Description: female child, nine years old, with Down Syndrome, congenital heart disease and prematurity, with significant weight loss and intermittent fever for six months. A week ago, she had an intensification of fever, productive cough and mild respiratory distress. RTPCR for SARS-CoV-2 detectable in nasopharynx swab, chest X-ray with diffuse alveolar infiltrate, chest $C T$ with consolidations, excavation, solid micronodules in a sprouting tree pattern mainly in the right upper and lower lobes. Molecular rapid test for Mycobacterium tuberculosis detectable in gastric lavage. After specific treatment, the patient progressed well and was discharged from the hospital after 72 hours without fever and improvement in her breathing pattern.

Discussion: despite the extensive pulmonary involvement, the patient did not require invasive ventilatory support and presented a satisfactory short-term outcome. Therefore, the relevance of the association of Tuberculosis and COVID-19 and other comorbidities in the pediatric age group still remains uncertain.

Key words Coronavirus, Tuberculosis, Coinfection, Pediatrics
\end{abstract}

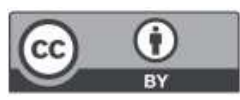




\section{Introduction}

In December 2019, a pneumonia caused by a new coronavirus, posteriorly identified as SARS-CoV-2, started in the city of Wuhan, China, resulting in the second pandemic of XXI century. The disease caused by this new infectious agent known as COVID-19. In Brazil, the first notification of a confirmed cased occurred in February 2020, and until September 19 of the same year, 4,582,240 cases and 136,532 deaths were confirmed.1,2

In children with COVID-19, fever and cough are the most common clinical manifestations, followed by myalgia, nasal congestion, coryza, odynophagia, cephalea, diarrhea and abdominal pain. Compared to adults, most diagnosed pediatric patients present mild symptoms, faster recovery and good prognosis. 1

Down syndrome determines immune deregulation, and thus, higher prevalence of autoimmune diseases, increase in hospitalization rates during respiratory viral infections and higher lethality rates by pneumonia and sepsis. So, besides the fact that pediatric age range presents, comparatively, less cases, children with Down Syndrome, due to the presence of comorbidities such as immunodeficiency, cardiovascular diseases, obesity and diabetes, are considered a risk population for severe COVID-19.

Specifically, immune dysregulation caused by trisomy of 21 chromosome may result in a exacerbated syndrome of liberation of cytokines, in relation to that observed in euploid population, thus, justifying additional monitoring and specialized care to this vulnerable population. 3,4

We also highlight that coinfection with Mycobacterium tuberculosis has been considered, until now, an important comorbidity, predictor of worse clinical outcomes. Besides that, within children, there is no sufficient scientific evidence to corroborate this affirmation, being prudent to emphasize social distancing and isolation of these patients, similarly of what is done with adults in order to mitigate transmissibility, and consequently the occurrence of possible severe cases. 5

\section{Description}

Nine years old child, female gender, from Fortaleza, State of Ceará, with Down syndrome and congenital heart disease (interatrial communication and patent ductus arteriosus, with surgical correction at 2 years old). Prematurity history (gestational age of 30 weeks) and extremely low birth weight $(800 \mathrm{~g})$, significant weight loss history and intermittent fever in six months. Approximately one week before hospitalization, referred intensification of fever, productive cough and mild respiratory distress, searching for medical care.

At admission time, she presented tachypnea, with mild respiratory distress, blood saturation between 89 and $92 \%$ in room air, needing oxygen supply via nasal catheter, diffuserhonchi at pulmonary auscultation. Onset of empirical treatment with oseltamivir, associated with ceftriaxone, oxacillin and azithromycin. Nonspecific laboratorial exams were requested, so as investigation of infection by SARS-CoV-2 via Real-Time Polymerase Chain Reaction nasopharyngeal swab (RT-PCR), with detectable result. Chest radiography evidenced diffuse alveolar infiltrate (Figure 1). At absence of clinical improvement, antimicrobial treatment was expanded with piperacillin-tazobactam, with a duration of 10 days.

High resolution CT (computed tomography) of the chest evidenced multiple confluent consolidations distributed predominantly in the bronchovascular bundles, with some sparse excavation focuses as well as countless solid micro-nodules, many of them with tree-in-bud pattern or rosette pattern, which affected more superior and inferior right lobes (Figure 2).

There was a report of contact with a brother who had pulmonary tuberculosis treated three years before. In face of that, rapid molecular test for Mycobacterium tuberculosis (GeneXpert MTB/RIF)was requested, whose result was detectable in two samples of gastric lavage.

After the onset of treatment with rifampicin, isoniazid and pyrazinamide, the patient exhibited clinical and respiratory standard improvement, and was discharged from hospital after 72 hours without fever, with orientations for returning and ambulatory follow-up.

\section{Discussion}

The World Health Organization emphasized that one of the main issues to be approached in relation to COVID-19 pandemic would be to understand risk factors for the severity of disease. Age over 65 years, diabetes and cardiovascular disease are referred as the main conditions associated with severe outcomes in this disease.6,7 Until now, however, there is no certainty regarding individuals under 18 years of age, which makes important the report of pediatric cases while definitive evidence in this way is not accumulated. 
Figure 1

Chest radiography in posterior-anterior position (A) and profile (B) in a 9 years old children with Down syndrome, coinfected by SARS-CoV-2 and Mycobacterium tuberculosis. Fortaleza, Ceará, 2020.

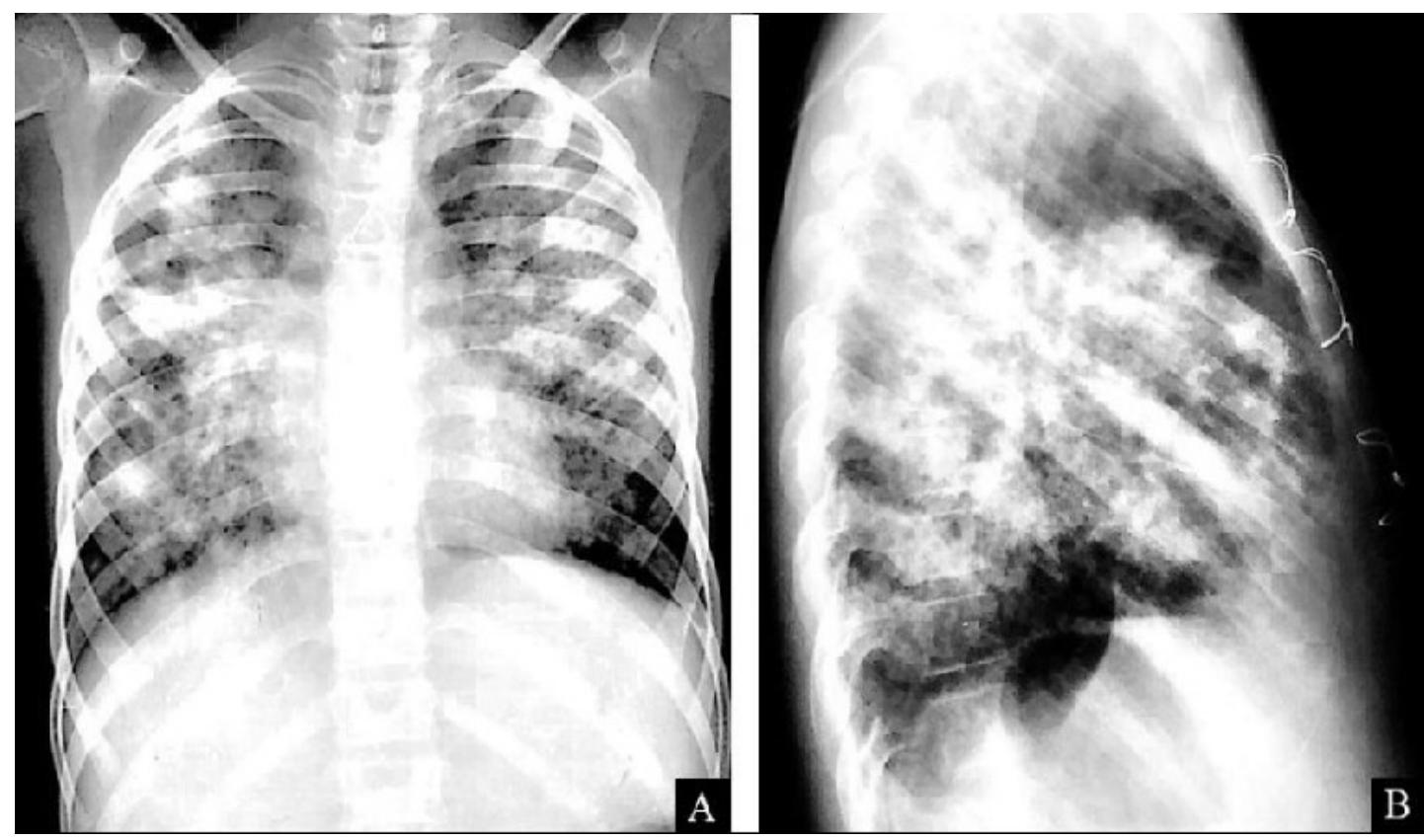

Note: Image evidencing extensive diffuse alveolar infiltrate.

\section{Figure 2}

High-resolution chest computed tomography in nine years old children with Down syndrome, coinfected by SARS-CoV2 and Mycobacterium tuberculosis. Fortaleza, Ceará, 2020.

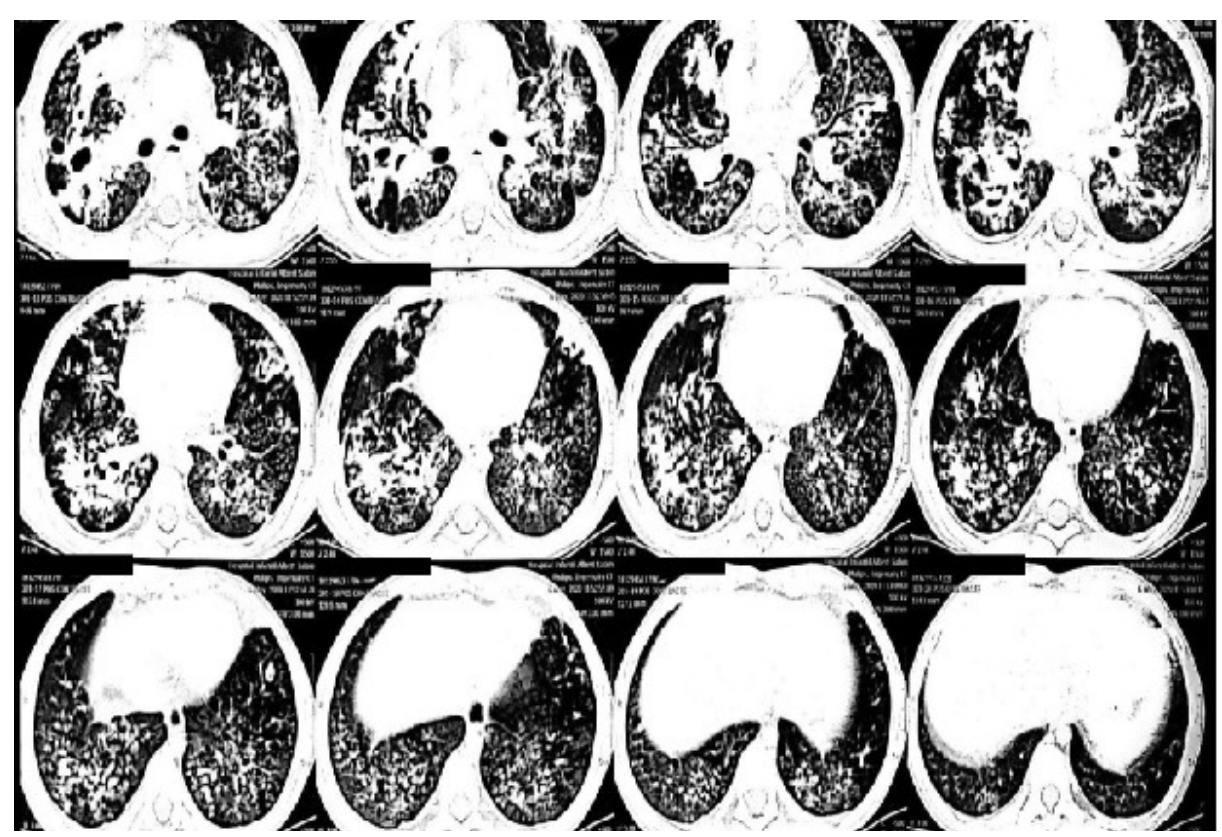

Note: Image evidencing multiple confluent consolidations in bronchovascular bundles, with some sparse excavation focuses as well as countless solid micro-nodules, many of them with tree-in-bud pattern or rosette pattern, which affected more the superior and inferior right lobes. 
The small rate of pediatric infections is not yet fully elucidated, which can be partially explained by the disease's physiopathology. Although scarce, studies related to profile of cytokines, subtypes of lymphocytes in pediatric patients, and consequently, children's immune system, denote a more appropriate response to the infectious agent, given that most of COVID-19 patients show normal lymphocyte count. 8

As cough and fever are common COVID-19 and tuberculosis symptoms, in the absence of competent anamnesis in the emergency context, usually restricted to the current patient's complaint and the evaluation of a rigorous epidemiological context, coinfection with Mycobacterium tuberculosis can be omitted. Notwithstanding, in opposition to the first, pulmonary tuberculosis is reflected by prolonged evolution. This was, initially, the situation of the child, which presented six months of fever and respiratory manifestations, besides weight loss, in which the suspicion of infection by mycobacterium stands as main diagnosis hypothesis.

It is possible that the context of the necessary social isolation due to the new disease and difficulty of access to routine consultations, and even emergency attendance, had favored the delay of tuberculosis diagnosis in this child, as well as in many other patients. We also highlight, as consequence, the decrease in quality of the treatment of disease, in general, with severe consequences to programs that are already in course. Besides, patients with tuberculosis generally present subjacent comorbidities and pulmonary damage that can make them prone to severe COVID-19.9

However, the patient presented satisfactory short-term evolution, even with the presumable additional risk represented by the immune alterations of Down syndrome. Although, it cannot be concluded that most patients would have the same outcome. It is also necessary the follow-up of the children after hospital discharge, in order to assure this outcome in middle and long term.

In a observational case-control study carried out by Chen et al., 1086 patients in suspicion of COVID19 were assessed in Shengyang, China, of which 36 were confirmed, and 13 (36.11\%) had positive IGRA (Interferon Gamma Release Assay) tests, three having active tuberculosis. The evaluation of percentage of severe and critical COVID-19 cases with tuberculosis coinfection was significantly higher than in the mild and moderate group. Seven of nine severe and critical cases presented coinfection with tuberculosis. These data infer that coinfection of SARS-CoV-2 and Mycobacterium tubercu- losis tends to lead to the development of more severe symptoms. Yet in this study, tuberculosis infection rates were considerably higher in COVID-19 patients than in patients with bacterial and viral pneumonias of other etiologies that were also evaluated, which suggests that Mycobacterium tuberculosis infection may be a specific risk factor for SARS-CoV-2 infection. Accordingly, individuals with latent or active tuberculosis may be more susceptible to new coronavirus, and the progression of the disease can be faster and more severe. 9

In Down syndrome, affected individuals present chronic auto-inflammation markers, including interferon hyperactivity, elevated level of inflammatory cytokines and chemokines and immune alterations. Nevertheless, it is not clear how these individuals may react to the new infection, so that the control, prevention and treatment recommendations remain the same as the general population. $3,4,11$

It is possible that, even with the two comorbidities, the patient did not show expected COVID-19 complications due to qualitative differentiations in the immune response of this age range compared to adults, mainly elderly, which developed differentiations in the level of expression of Angiotensinconverting enzyme 2, cell receptor for the viral infection. Other factors include the increase of proinflammatory cytokines that control neutrophil functions due to aging, which has been correlated to severity of the new disease, besides the simultaneous presence of other virus in the respiratory tract, more common in children, establishing a disadvantageous competition for the new coronavirus, or even lymphocyte and cytokines imbalance described in patients with Down syndrome.3,10

Thus, this report alerts that coinfection with Mycobacterium tuberculosisshould not be disregarded in patients with COVID-19, due to the possibility of aggravating the evolution of one or both conditions, as literature suggests until now. Notwithstanding, despite the significant pulmonary impairment, in opposition to what was expected, the patient presented satisfactory outcome in short-term, without necessity of invasive ventilation support.

Therefore, the relevance of association of tuberculosis and COVID-19 and other comorbidities in the pediatric age range remain unclear. It is important to highlight that, even in social isolation context, the access to tuberculosis treatment should be assured, which requires regularity, as well as diagnosis investigation of patients with prolonged respiratory symptoms and the follow-up of children with special needs. 


\section{Author's contribution}

Stefanuto PPG performed planning, data collection, writing and review of the manuscript. Fernandes CJS supported the planning and data collection, writing of the discussion of the reported case, besides the review of the article. Cruz CG accompanied and supported the review of the manuscript, as well as

\section{References}

1. She J, Liu L, Liu W. COVID-19 epidemic: Disease characteristics in children. J Med Virol. 2020; 92 (7): 747-54.

2. Brasil. Ministério da Saúde. Secretaria de Vigilância em Saúde. Boletim Epidemiológico Especial - Doença pelo Coronavírus COVID-19 32, Semana Epidemiológica 38 (13 a 19 de setembro de 2020). [acesso 20 set 2020]. Disponível em:

http://portalarquivos2.saude.gov.br/images/pdf/2020/Septe mber/ 23/Boletim-epidemiologico-COVID-32-final23.09_18h30.pdf

3. Espinosa J. Down Syndrome and COVID-19: A Perfect Storm? Cell Rep Med. 2020; 1 (2): 1-8.

4. Callea M, Cammarata-Scalisi F, Galeotti A, Villani A Valentini D. COVID-19 and Down syndrome. Acta Paediatr. 2020; 109: 1901-2.

5. Maciel E, Gonçalves Júnior E, Dalcolmo M. Tuberculose e coronavírus: o que sabemos? Epidemiol Serv Saúde. 2020 29 (2): 128

6. United States. Department of Health and Human Services. Centers for Disease Control and Prevention. Severe Outcomes Among Patients with Coronavirus Disease 2019 (COVID-19) - United States, February 12-March 16 2020. MMWR Morb Mortal Wkly Rep 2020; 69:343-346. [acesso em 10 jul 2020]. Disponível em: http://dx.doi.org/10.15585/mmwr.mm6912e2 supported the case description. Leite RD accompanied and supported the review of the manuscript, as well as the writing of discussion and description of the article. Tavares LVS accompanied and helped the manuscript review, as well as the writing of the introduction and description of the article.

7. Xu P, Tian R, Luo S, Zu Z, Fan B, Wang X, Xu K, Wang J, Zhu, J, Shi J, Chen F, Wan B, Yan Z, Wang R, Chen W, Fan W, Zhang C, Lu M, Sun Z, Zhou C, Zhang L, Xia F, Qi L, Zhang W, Zhong J, Liu X, Zhang Q, Lu G, Zhang L. Risk Factors for Adverse Clinical Outcomes With COVID-19 in China: A Multicenter, Retrospective, Observational Study. Theranostics. 2020; 10 (14): 6372-83.

8. Silva J, Argentino A, Dulaba L, Bernardelli R, Campiolo, E. COVID-19 em pediatria: um panorama entre incidência e mortalidade. Resid Pediatr. 2020; 0 (0): 383.

9. Togun T, Kampmann B, Stoker N, Lipman M. Anticipating the Impact of the COVID-19 Pandemic on TB Patients and TB Control Programmes. Ann Clin Microbiol Antimicrob. 2020; 19 (1): 21 .

10. Yu Chen, Yaguo Wang, Joy Fleming, Yanhong Yu, Ye Gu, Chang Liu, Lichao Fan, Xiaodan Wang, Moxin Cheng, Lijun Bi, Yougyu Liu. Active or latent tuberculosis increases susceptibility to COVID-19 and disease severity. MedrxivmedRxiv. 2020; 20 (3): 795.

11. Raskin S, Toralles M, Martins A, Ribeiro E, Paiva I, Galera M, Silva R. COVID-19 e Síndrome de Down - Nota de Alerta. Soc Bras Pediatr. 2020; 2: 1-9.

Received on September 29, 2020

Approved on December 29, 2020 